\title{
EDITORIAL
}

\section{El carcinoma epitelial de ovario}

Si bien la incidencia de carcinoma de ovario no es tan elevada, el problema radica en la alta tasa de mortalidad que presenta; en USA es la primera causa de muerte por cáncer genital femenino y en nuestro pais es superado en mortalidad por el carcinoma de cuello uterino. La probabilidad de presentar un cáncer ovárico a lo largo de su vida para una mujer americana es de $1 / 70$, es más frecuente en mujeres postmenopáusicas y en aquellas con historia familiar de carcinoma de ovario, y aproximadamente el $1 \%$ de estos tumores ocurren como parte del sindrome de cáncer hereditario.

Se han propuesto varias estrategias de tamizaje para ser utilizadas en la población general, pero hasta ahora los elevados costos de su implementación no han convencido a los expertos en relación con las reducciones que se obtendrian en morbilidad y mortalidad.

El CA-125 es un marcador que se encuentra elevado en cerca del 80 por ciento de los tumores ováricos epiteliales malignos pero sólo es positivo en cerca del 20 por ciento de estados uno y además se encuentra elevado en algunos otros tumores benignos como los endometriomas y en varios procesos benignos como los que comprometen el peritoneo.

El ultrasonido transvaginal ha ganado terreno especialmente si se aplica a poblaciones de mayor riesgo y si bien su sensibilidad es alta, su especificidad y el valor predictivo positivo son moderados de acuerdo con. los investigadores; siembargo cuando se afinan más los parámetros morfológicos de evaluación se obtienen mejores resultados. Los estudios actualmente disponibles indican que el ultrasonido transvaginal permite detectar tumores ováricos en estadios más iniciales con la consiguiente disminución de la mortalidad; lo que está por demostrar es si un ultrasonido transvaginal anual reduce la mortalidad en la población general y esto sólo se sabrá luego de realizar estudios multicéntricos válidos. Los estudios con Doppler color como complemento al ultrasonido son un poco más costosos y aunque son prometedores, están lejos de ser utilizados rutinariamente. El TAC y la RNM son estudios considerados más como de extensión y estadificación que de diagnóstico.

Proyectos como el de la Universidad de Kentucky han insinuado la implementación de la Laparoscopia como método complementario al Ca-125 y al Ultrasonido para diagnóstico y eventual tratamiento quirúrgico inmediato de lesiones malignas en Estados Uno, manejo que justifican con una hipotética disminución de costos en relación con lo que valdría el tratamiento de un carcinoma avanzado, incluidos la cirugía y la quimioterapia; en ese programa piloto de investigación han encontrado un carcinoma por cada mil pacientes postmenopáusicas asintomáticas sometidas a screening.

Alrededor del $18 \%$ de las pacientes con cáncer de ovario han sido sometidas a histerectomía previa con conservación de uno o ambos ovarios, circunstancia que ha permitido que algunos autores defiendan desde la óptica oncológica la ooforectomía profiláctica previo consentimiento informado en pacientes mayores de 40 años que requieran histerectomía por otra causa, con lo cual se lograría reducir de manera significativa la incidencia de carcinoma ovárico. Para las pacientes con historia de cáncer ovárico hereditario la ooforectomía profiláctica se ha propuesto para mayores de 35 años si la paridad está satisfecha. Así mismo las pacientes postmenopáusicas que tienen indicación para cirugia abdominal deben ser sometidas a ooforectomía profiláctica.

La cirugía laparoscópica ha tenido cada vez más aceptación en el abordaje de masas pélvicas y ante lesiones con sospecha clínica o paraclínica de malignidad se debe contar con el concurso del ginecólogo oncólogo puesto que en relación con la sobrevida está bien definido que quien realiza el primer procedimiento quirúrgico es quien define el pronóstico de la paciente, consideración tan válida como cuando se enfrenta un caso con cirugía abdominal abierta, asi se trate de una lesión de bajo potencial de malignidad o un estado aparentemente inicial, los cuales requieren una exploración muy completa del abdomen y del retroperitoneo en busca de enfermedad microscópica, para considerar o no la necesidad de un tratamiento complementario; si no se actúa correctamente la paciente tendrá que ser sometida a otra exploración quirúrgica o a una conducta inadecuada. Desde luego que estos procedimientos no sólo exigen que sean realizados por profesionales que tengan el entrenamiento y la experiencia necesarias para tal fin, sino que deben ser llevados a cabo en instituciones que dispongan de la infraestructura tecnológica suficiente como la posibilidad de practicar la biopsia por congelación, banco de sangre y poder resolver complicaciones agudas, entre otras.

La utilización de la biopsia por congelación cuando está indicada, la exploración completa y la mayor citoreducción posible para optimizar la acción de la quimioterapia son condiciones requeridas en procura de una mejor sobrevida. 
En las pasadas dos décadas el manejo complementario a la cirugía cuando es necesario en los tumores epiteliales malignos de ovario se ha realizado con esquemas combinados con base en derivados del platino, los cuales pueden utilizarse aún para la recidiva cuando ésta se presenta luego de 6 meses del tratamiento inicial. Si la recidiva ocurre antes de los 6 meses se considera que el caso es resistente a los derivados del platino y si es necesario deberá utilizarse una segunda línea de tratamiento diferente a los platinos.

Durante los últimos años se han introducido algunos medicamentos de segunda línea como el taxol, el topotecán y la gemcitavina para tratamientos de rescate de los casos recidivantes y ya existen protocolos en los cuales se utilizan como esquemas de primera línea los dos primeros, especialmente la combinación platino-taxol con el argumento de potenciar los efectos puesto que tienen mecanismo de acción diferentes, en búsqueda de una mejor respuesta inicial puesto que los resultados obtenidos en los tratamientos de las recidivas, las persistencias o en los casos refractarios no son muy estimulantes.

El gran dilema para un medio como el nuestro se presenta precisamente cuando debemos enfrentarnos a la recidiva o a la persistencia y tenemos que decidir entre un costoso esquema de segunda linea con el cual obtendremos respuestas totales cercanas al $20 \%$ con prolongación de la sobrevida por unos pocos meses en la mayoría de los casos o nos decidimos por esquemas menos onerosos con monoagentes como el etopósido, la hexametilmelamina, o el tamoxifén, especialmente en aquellas pacientes con recidiva manifestada en el marcador pero asintomáticas, grupo en el cual a veces la observación clínica puede ser lo más indicado. El acetato de megestrol es una buena alternativa ante la enfermedad incurable para el manejo de la anorexia y otros sintomas afines, los cuales además deben abordarse con una bien dirigida terapia paliativa. La actitud que asuma cada grupo tratante debe ajustarse a las condiciones del paciente en primera instancia y luego a las condiciones del medio sin desconocer el impacto emocional, social y económico que representan las decisiones asumidas; y finalmente lo ideal es que todos estos manejos puedan desarrollarse dentro de protocolos multidisciplinarios y ojalá multi institucionales de investigación. Igual enfoque debe dársele a la utilización de la Operación de Segunda Mirada puesto que no ha tenido un verdadero impacto en la sobrevida de las pacientes con carcinoma de ovario, sinembargo racionalmente utilizada y siguiendo parámetros claros puede ser benéfica. Desde luego que una Segunda Mirada implica la necesidad de disponer de una segunda línea de tratamiento.

GILBERTO MARTINEZ MORALES Ginecólogo Oncólogo 